\title{
General Type-2 Fuzzy Topological Spaces
}

\author{
Munir Abdul Khalik AL-Khafaji, Mohammed Salih Mahdy Hussan \\ Department of Mathematics, College of Education, AL-Mustinsiryah University, Baghdad, Iraq \\ Email: mnraziz@yahoo.com, mssm_1975@yahoo.com
}

How to cite this paper: AL-Khafaji, M.A.K. and Hussan, M.S.M. (2018) General Type-2 Fuzzy Topological Spaces. Advances in Pure Mathematics, 8, 771-781.

https://doi.org/10.4236/apm.2018.89047

Received: August 7, 2018

Accepted: September 18, 2018

Published: September 21, 2018

Copyright $\odot 2018$ by authors and Scientific Research Publishing Inc. This work is licensed under the Creative Commons Attribution International License (CC BY 4.0).

http://creativecommons.org/licenses/by/4.0/

\begin{abstract}
In this paper, a presented definition of type- 2 fuzzy sets and type-2 fuzzy set operation on it was given. The aim of this work was to introduce the concept of general topological spaces were extended in type-2 fuzzy sets with the structural properties such as open sets, closed sets, interior, closure and neighborhoods in topological spaces were extended to general type-2 fuzzy topological spaces and many related theorems are proved.
\end{abstract}

\section{Keywords}

Type-2 Fuzzy Set, Interval Type-2 Fuzzy Topological Space, General Type-2 Fuzzy Topological Spaces, Type-2 Fuzzy Open Sets, Type-2 Fuzzy Closed Sets, Type-2 Fuzzy Interior, Type-2 Fuzzy Closure, Neighborhood of a Type-2 Fuzzy Set

\section{Introduction}

The fuzzy set theory proposed by Zadeh [1] extended the classical notion of sets and permitted the gradual assessment of membership of elements in a set [2]. After introducing the notion of fuzzy sets and fuzzy set operations, several attempts have been made to develop mathematical structures using fuzzy set theory. In 1968, chang [3] introduced fuzzy topology which provides a natural framework for generalizing many of the concepts of general topology to fuzzy topological spaces and its development can be found in [3]. The concept of a type-2 fuzzy set as extension of the concept of an ordinary fuzzy set (henceforth called a type-1 fuzzy set) in which the membership function falls into a fuzzy set in the interval [0,1], [2] [4]. Many scholars have conducted research on type-2 fuzzy set and their properties, including Mizumoto and Tanaka [5], Mendel [6], Karnik and Mendel [4] and Mendel and John [7]. Type-2 fuzzy sets are called "fuzzy", so, it could be called fuzzy set [6]. In [6] Mendel was introduced the concept of an interval type-2 fuzzy set. Type-2 fuzzy sets have also been widely 
applied to many fields with two parts general type-2 fuzzy set and interval type- 2 fuzzy sets. The interval type-2 fuzzy topological space introduced by [2]. Because the interval type-2 fuzzy set, as a special case of general type-2 fuzzy sets, and general type- 2 fuzzy sets may be better that the interval type-2 fuzzy sets to deal with uncertainties and because general type- 2 fuzzy sets can obtain more degrees of freedom [8], we introduce general type-2 fuzzy topological spaces. The paper is organized as follows. Section 2 is the preliminary section which recalls definitions and operations to gather with some properties type-2 fuzzy sets. In Section 3 , we introduce the definition of general type-2 fuzzy topology and some of its structural properties such as type-2 fuzzy open sets, type-2 fuzzy closed sets, type-2 fuzzy interior, type- 2 fuzzy closure and neighborhood of a type-2 fuzzy set are studied.

\section{Preliminaries}

In this section, we recall the preliminaries of type- 2 fuzzy sets, define type- 2 fuzzy and some important associated concepts from [7] [9] and throughout this paper, let $X$ be anon empty set and $I$ be closed unit interval, i.e., $I=[0,1]$.

Definition 1 [7] [9]. Let $X$ be a finite and non empty set, which is referred to as the universe a type- 2 fuzzy set, denoted by $\tilde{\tilde{A}}$ is characterized by a type- 2 memberships function $\mu_{\tilde{\tilde{A}}}(x, u)$, as

$\mu_{\tilde{\tilde{A}}}: X \times[0,1] \rightarrow[0,1]^{J_{x}}\left(J_{x} \subseteq[0,1]\right)$, where $x \in X$ and $u \in J_{x}$, that is

$\tilde{\tilde{A}}=\left\{\left((x, u), \mu_{\tilde{\tilde{A}}}(x, u)\right):\right.$ where $x \in X$ and $u \in J_{x} \subseteq[0,1]$, where $\left.0 \leq \mu_{\tilde{\tilde{A}}}(x, u) \leq 1\right\}$

$\tilde{\tilde{A}}$ can also be expressed as

$$
\begin{aligned}
\tilde{\tilde{A}} & =\sum_{x \in X} \sum_{u \in J x} \mu_{\tilde{\tilde{A}}}(x, u) /(x, u) \\
& =\sum_{x \in X} \sum_{u \in J x} f_{x}(u) / u / x, J_{x} \subseteq[0,1]
\end{aligned}
$$

where $f_{x}(u)=\mu_{\tilde{\tilde{A}}}(x, u)$ an $\sum \sum$ denotes union over all admissible $x$ and $u$ for continuous universes of discourse, $\sum$ is replaced by $\int$. The class of all type-2 fuzzy sets of the universe $X$ denoted by $\tilde{\mathbb{F}}_{T_{2}}(X)$.

Definition 2 [2] [7]. A vertical slice, denoted $\mu_{\tilde{\tilde{A}}}\left(x^{\prime}\right)$, of $\tilde{\tilde{A}}$, is the intersection between the two-dimensional plane whose axes are $u$ and $\mu_{\tilde{\tilde{A}}}\left(x^{\prime}, u\right)$ and the three-dimensional type-2membership function $\tilde{\tilde{A}}$, i.e., $\mu_{\tilde{\tilde{A}}}\left(x^{\prime}\right)=\mu_{\tilde{\tilde{A}}}\left(x=x^{\prime}, u\right)=\sum_{u \in J_{x^{\prime}}} f_{x^{\prime}}(u) / u, J_{x^{\prime}} \subseteq I \quad$ in which $\quad 0 \leq f_{x^{\prime}}(u) \leq 1 . \quad \tilde{\tilde{A}}$ can also be expressed as follows: $\tilde{\tilde{A}}=\left\{\left(x, \mu_{\tilde{\tilde{A}}}(x)\right): \forall x \in X\right\}$ or as following

$$
\begin{aligned}
\tilde{\tilde{A}} & =\sum_{x \in X} \sum_{u \in J x} \mu_{\tilde{\tilde{A}}}(x) /(x) \\
& =\sum_{x \in X} \sum_{u \in J x} f_{x}(u) / u / x, J_{x} \subseteq[0,1]
\end{aligned}
$$

The vertical slice, $\mu_{\tilde{\tilde{A}}}\left(x^{\prime}\right)$ is also called the secondary membership function, and its domain is called the primary membership of $x$, which is denoted by $J_{X}$ where $J_{X} \subseteq I$ for any $x \in X$. The amplitude of a secondary membership 
function is called the secondary grade.

When configuring any type-2 fuzzy topological structures we must present some special types of type-2 fuzzy sets.

Definition 3 [5] [8]. ( Type-2 fuzzy universe set).

A type-2 fuzzy universe set, denoted $\tilde{\tilde{X}}$, such that

$$
\tilde{\tilde{X}}=\sum_{x \in X} \sum_{u \in[1,1]} 1 / u / x
$$

Definition 4 [5] [8]. ( Type-2 fuzzy empty set)

A type-2 fuzzy empty set, denoted $\tilde{\tilde{\varnothing}}$, such that

$$
\tilde{\varnothing}=\sum_{x \in X} \sum_{u \in[0,0]} 1 / u / x
$$

Definition 5 [6]. (Interval type-2 fuzzy set).

When all the secondary grades of types $\tilde{\tilde{A}}$ are equal to 1 , that is $\mu_{\tilde{\tilde{A}}}(x, u)=1$ for all $x \in X$ and for all $u \in J_{x} \subseteq[0,1], \tilde{\tilde{A}}$ is as an Interval type-2 fuzzy set.

Operation of Types-2 fuzzy sets 6. Consider two type-2 fuzzy sets, $\tilde{\tilde{A}}$ and $\tilde{\tilde{B}}$, in a universe $X$. Let $\mu_{\tilde{\tilde{A}}}(x)$ and $\mu_{\tilde{\tilde{B}}}(x)$ be the membership grades of these two sets, which are represented for each $x \in X, \mu_{\tilde{\tilde{A}}}(x)=\sum_{u \in J_{x}^{u}} f_{x}(u) / u$ and $\mu_{\tilde{\tilde{B}}}(x)=\sum_{w \in J_{x}^{w}} g_{x}(w) / w$, respective, where $u \in J_{x}^{u}, w \in J_{x}^{w}$ indicate the primary memberships of $x$ and $f_{x}(u), g_{x}(w) \in[0,1]$ indicate the secondary memberships (grades) of $x$. The membership grades for the union, intersection and complement of the type-2 fuzzy sets $\tilde{\tilde{A}}$ and $\tilde{\tilde{B}}$ have been defined as follows [5].

Containment:

$\tilde{\tilde{A}}$ is a subtype-2 fuzzy set of $\tilde{\tilde{B}}$ denoted $\tilde{\tilde{A}} \subseteq \tilde{\tilde{B}}$ if $u \leq w$ and $f_{x}(u) \leq g_{x}(w)$ for every $x \in X$.

\section{Equality:}

$\tilde{\tilde{A}}$ and $\tilde{\tilde{B}}$ are type-2 fuzzy sets are equal, denoted $\tilde{\tilde{A}}=\tilde{\tilde{B}}$ if $u=w$ and $f_{x}(u)=\mu_{\tilde{\tilde{A}}}(x, u)=g_{x}(w)=\mu_{\tilde{\tilde{B}}}(x, w)$ for every $x \in X$.

Union of two type-2 fuzzy sets:

$$
\begin{aligned}
\tilde{\tilde{A}} \cup \tilde{\tilde{B}} \Leftrightarrow \mu_{\tilde{\tilde{A}} \tilde{\tilde{B}}}(x) & =\sum_{u \in J_{x}^{u}} \sum_{w \in J_{x}^{w}} f_{x}(u) \star g_{x}(w) /(u \vee w) \\
& \equiv \mu_{\tilde{\tilde{A}}}(x) \sqcup \mu_{\tilde{\tilde{B}}}(x), \quad x \in X
\end{aligned}
$$

Intersection of two type-2 fuzzy sets:

$$
\begin{aligned}
\tilde{\tilde{A}} \cap \tilde{\tilde{B}} \Leftrightarrow \mu_{\tilde{\tilde{A}} \cap \tilde{\tilde{B}}}(x) & =\sum_{u \in J_{x}^{u}} \sum_{w \in J_{x}^{w}} f_{x}(u) \star g_{x}(w) /(u \vee w) \\
& \equiv \mu_{\tilde{\tilde{A}}}(x) \sqcap \mu_{\tilde{\tilde{B}}}(x), \quad x \in X
\end{aligned}
$$

Complement of a type-2 fuzzy set:

$$
\sim \tilde{\tilde{A}}=\mu_{\sim \tilde{\tilde{A}}}(x)=\sum_{u \in J_{x}^{u}} f_{x}(u) /(1-u) \equiv \neg \mu_{\tilde{\tilde{A}}}(x), x \in X
$$

Where $\vee$ represent the max $\mathrm{t}$-conorm and $\star$ represent a t-norm. The summation indicate logical unions. We refer to the operations $\sqcup, \sqcap$ and $\neg$ as join, meet and negation respectively and $\mu_{\tilde{\tilde{A}} \cup \tilde{\tilde{B}}}(x), \mu_{\tilde{\tilde{A}} \wedge \tilde{\tilde{B}}}(x), \mu_{\tilde{\tilde{A}}}(x)$ and $\mu_{\tilde{\tilde{B}}}(x)$ are the secondary membership functions and all are type-1 fuzzy sets. If 
$\mu_{\tilde{\tilde{A}}}(x)$ and $\mu_{\tilde{\tilde{B}}}(x)$ have continuous domains, then the summations in 3, 4 and 5 are replaced by integrals.

Example 7: Let $X=\left\{x_{1}, x_{2}, x_{3}\right\}$ be anon empty set, and let $\tilde{\tilde{A}}$ and $\tilde{\tilde{B}}$ are type-2 fuzzy sets over the same universe $X$.

$$
\begin{aligned}
& \tilde{\tilde{A}}=\left\{\left(\left(x_{1}, 0.1\right), 0.3\right),\left(\left(x_{1}, 0.5\right), 1\right),\left(\left(x_{2}, 0.5\right), 1\right),\left(\left(x_{2}, 0.6\right), 0.3\right),\left(\left(x_{3}, 0.8\right), 1\right)\right\} \\
& \tilde{\tilde{B}}=\left\{\left(\left(x_{1}, 0.1\right), 0.7\right),\left(\left(x_{1}, 0.2\right), 1\right),\left(\left(x_{2}, 0.6\right), 1\right),\left(\left(x_{3}, 0.5\right), 0.6\right),\left(\left(x_{3}, 0.9\right), 1\right)\right\} \\
& \tilde{\tilde{A}} \cup \tilde{\tilde{B}} \text { for } x=x_{1} \text { to get }
\end{aligned}
$$$$
\mu_{\tilde{\tilde{A}} \cup \tilde{B}}\left(x_{1}\right)=\frac{0.3 \wedge 0.7}{0.1 \vee 0.1}+\frac{0.3 \wedge 1}{0.1 \vee 0.2}+\frac{1 \wedge 0.7}{0.5 \vee 0.1}+\frac{1 \wedge 1}{0.5 \vee 0.2}
$$$$
=\frac{0.3}{0.1}+\frac{0.3}{0.2}+\frac{0.7}{0.5}+\frac{1}{0.5}=\{(0.1,0.3),(0.2,0.3),(0.5, \max \{0.7,1\})\}
$$

$\tilde{\tilde{A}} \cup \tilde{\tilde{B}}$ for $x=x_{1},\left\{\left(\left(x_{1}, 0.1\right), 0.3\right),\left(\left(x_{1}, 0.2\right), 0.3\right),\left(\left(x_{1}, 0.5\right), 1\right)\right\}$

$\tilde{\tilde{A}} \cup \tilde{\tilde{B}}$ for $x=x_{2}$ to get

$\mu_{\tilde{\tilde{A}} \cup \tilde{B}}\left(x_{2}\right)=\frac{1 \wedge 1}{0.5 \vee 0.6}+\frac{0.3 \wedge 1}{0.6 \vee 0.6}=\frac{1}{0.6}+\frac{0.3}{0.6} \Rightarrow\{(0.6, \max \{1,0.3\})\}$

$\tilde{\tilde{A}} \cup \tilde{\tilde{B}}$ for $x=x_{2} \Rightarrow\left\{\left(\left(x_{2}, 0.6\right), 1\right)\right\}$

$\tilde{\tilde{A}} \cup \tilde{\tilde{B}}$ for $x=x_{3}$ to get

$\mu_{\tilde{\tilde{A}} \cup \tilde{B}}\left(x_{3}\right)=\frac{1 \wedge 0.6}{0.8 \vee 0.5}+\frac{1 \wedge 1}{0.8 \vee 0.9}=\frac{0.6}{0.8}+\frac{1}{0.9}=\{(0.8,0.6),(0.9,1)\}$

$\tilde{\tilde{A}} \cup \tilde{\tilde{B}}$ for $x=x_{3},\left\{\left(\left(x_{3}, 0.8\right), 0.6\right),\left(\left(x_{3}, 0.9\right), 1\right)\right\}$

$\tilde{\tilde{A}} \cup \tilde{\tilde{B}}=\left\{\left(\left(x_{1}, 0.1\right), 0.3\right),\left(\left(x_{1}, 0.2\right), 0.3\right),\left(\left(x_{1}, 0.5\right), 1\right),\left(\left(x_{2}, 0.6\right), 1\right)\right.$,

$$
\left.\left(\left(x_{3}, 0.8\right), 0.6\right),\left(\left(x_{3}, 0.9\right), 1\right)\right\}
$$

$\tilde{\tilde{A}} \cap \tilde{\tilde{B}}$ for $x=x_{1}$ to get

$$
\begin{aligned}
\mu_{\tilde{\tilde{A}} \cap \tilde{\tilde{B}}}\left(x_{1}\right) & =\frac{0.3 \wedge 0.7}{0.1 \wedge 0.1}+\frac{0.3 \wedge 1}{0.1 \wedge 0.2}+\frac{1 \wedge 0.7}{0.5 \wedge 0.1}+\frac{1 \wedge 1}{0.5 \wedge 0.2} \\
& =\frac{0.3}{0.1}+\frac{0.3}{0.1}+\frac{0.7}{0.1}+\frac{1}{0.2}=\{(0.1, \max \{0.3,0.3,0.7\}),(0.2,1)\}
\end{aligned}
$$

$\tilde{\tilde{A}} \cap \tilde{\tilde{B}}$ for $x=x_{1},\left\{\left(\left(x_{1}, 0.1\right), 0.7\right),\left(\left(x_{1}, 0.2\right), 1\right)\right\}$

$\tilde{\tilde{A}} \cap \tilde{\tilde{B}}$ for $x=x_{2}$ to get

$\mu_{\tilde{\tilde{A}} \cap \tilde{\tilde{B}}}\left(x_{2}\right)=\frac{1 \wedge 1}{0.5 \wedge 0.6}+\frac{0.3 \wedge 1}{0.6 \wedge 0.6}=\frac{1}{0.5}+\frac{0.3}{0.6} \Rightarrow\{(0.5,1),(0.6,0.3)\}$

$\tilde{\tilde{A}} \cap \tilde{\tilde{B}}$ for $x=x_{2},\left\{\left(\left(x_{2}, 0.5\right), 1\right),\left(\left(x_{2}, 0.6\right), 0.3\right)\right\}$

$\tilde{\tilde{A}} \cap \tilde{\tilde{B}}$ for $x=x_{3}$ to get

$\mu_{\tilde{\tilde{A}} \cap \tilde{\tilde{B}}}\left(x_{3}\right)=\frac{1 \wedge 0.6}{0.8 \wedge 0.5}+\frac{1 \wedge 1}{0.8 \wedge 0.9}=\frac{0.6}{0.5}+\frac{1}{0.8} \Rightarrow\{(0.5,0.6),(0.8,1)\}$

$\tilde{\tilde{A}} \cap \tilde{\tilde{B}}$ for $x=x_{3},\left\{\left(\left(x_{3}, 0.5\right), 0.6\right),\left(\left(x_{3}, 0.8\right), 1\right)\right\}$ 


$$
\begin{aligned}
& \tilde{\tilde{A}} \cap \tilde{\tilde{B}}=\left\{\left(\left(x_{1}, 0.1\right), 0.7\right),\left(\left(x_{1}, 0.2\right), 1\right),\left(\left(x_{2}, 0.5\right), 1\right),\left(\left(x_{2}, 0.6\right), 0.3\right),\right. \\
&\left.\left(\left(x_{3}, 0.5\right), 0.6\right),\left(\left(x_{3}, 0.8\right), 1\right)\right\}
\end{aligned}
$$

The complement of a type-2 fuzzy set $\tilde{\tilde{A}}$ is

$$
\begin{aligned}
& \sim \tilde{\tilde{A}}=\mu_{\sim \tilde{\tilde{A}}}(x)=\sum_{u \in J_{x}^{u}} f_{x}(u) /(1-u) \\
& \equiv \neg \mu_{\tilde{\tilde{A}}}(x), x \in X \\
&=\left\{\left(\left(x_{1}, 0.9\right), 0.3\right),\left(\left(x_{1}, 0.5\right), 1\right),\left(\left(x_{2}, 0.5\right), 1\right),\left(\left(x_{2}, 0.4\right), 0.3\right),\left(\left(x_{3}, 0.2\right), 1\right)\right\} .
\end{aligned}
$$

Operations under collection of type-2 fuzzy sets 8: Let $\left\{\tilde{\tilde{A}}_{i}: i \in \mathbb{N}\right\}$ be an arbitrary collection of type-2 fuzzy sets subset of $X$ such that $\mathbb{N}$ is countable set, operation are possible under an arbitrary collection of type-2 fuzzy sets.

1) The union $\cup_{i \in \mathbb{N}} \tilde{\tilde{A}}_{i}$ is defined as

$$
\left[\cup_{i \in \mathcal{N}} \tilde{\tilde{A}}_{i}\right](x)=\sum_{x \in X} \sum_{u \in J_{x}^{u}} \frac{\wedge_{i \in \mathcal{N}}\left(f_{x}(u)\right)_{i}}{\vee_{i \in \mathcal{N}}(u)_{i}}
$$

2) The intersection $\cap_{i \in \mathbb{N}} \tilde{\tilde{A}}_{i}$ is defined as

$$
\left[\cap_{i \in \mathbb{N}} \tilde{\tilde{A}}_{i}\right](x)=\sum_{x \in X} \sum_{u \in J_{x}^{u}} \frac{\wedge_{i \in \mathcal{N}}\left(f_{x}(u)\right)_{i}}{\wedge_{i \in \mathcal{N}}(u)_{i}}
$$

Proposition 9: Let $\left\{\tilde{\tilde{A}}_{i}: i \in \mathbb{N}\right\}$ be an arbitrary collection of type-2 fuzzy sets subset of $X$ such that $\mathbb{N}$ is countable set and $\tilde{\tilde{B}}$ be another type-2 fuzzy set of $X$, then
1) $\tilde{\tilde{B}} \cap\left[\cup_{i \in \mathbb{N}} \tilde{\tilde{A}}_{i}\right]=\cup_{i \in \mathbb{N}}\left(\tilde{\tilde{B}} \cap \tilde{\tilde{A}}_{i}\right)$.
2) $\tilde{\tilde{B}} \cup\left[\cap_{i \in \mathbb{N}} \tilde{\tilde{A}}_{i}\right]=\cap_{i \in \mathbb{N}}\left(\tilde{\tilde{B}} \cup \tilde{\tilde{A}}_{i}\right)$.
3) $1-\left[\cup_{i \in \mathbb{N}} \tilde{\tilde{A}}_{i}\right]=\cap_{i \in \mathbb{N}}\left(1-\tilde{\tilde{A}}_{i}\right)$.
4) $1-\left[\cap_{i \in \mathbb{N}} \tilde{\tilde{A}}_{i}\right]=\cup_{i \in \mathbb{N}}\left(1-\tilde{\tilde{A}}_{i}\right)$.

\section{General Type-2 Fuzzy Topological Space}

In this section we introduced the concept general type-2 fuzzy topology.

Definition 1: Let $\tilde{\tilde{F}}$ be the collection of type-2 fuzzy set over $X$; then $\tilde{\tilde{F}}$ is said to be general type-2 fuzzy topology on $X$ if

1) $\tilde{\tilde{\varnothing}}, \tilde{\tilde{X}} \in \tilde{\tilde{F}}$

2) $\tilde{\tilde{A}} \cap \tilde{\tilde{B}} \in \tilde{\tilde{F}}$ for any $\tilde{\tilde{A}}, \tilde{\tilde{B}} \in \tilde{\tilde{F}}$.

3) $\cup_{i \in \mathbb{N}} \tilde{\tilde{A}}_{i} \in \tilde{\tilde{\mathfrak{F}}}$ for any $\tilde{\tilde{A}}_{i} \in \tilde{\tilde{\mathfrak{F}}}, \mathbb{N}$ countable set.

The pair $(X, \tilde{\tilde{F}})$ is called general type-2 fuzzy topological space over $X$.

Remark 2: Let $(X, \tilde{\tilde{F}})$ be general type-2 fuzzy topological space over $X$; then the members of $\tilde{\tilde{F}}$ are said to be type-2 fuzzy open set in $X$ and a type- 2 fuzzy 
set $\tilde{\tilde{A}}$ is said to be a type-2 fuzzy closed set in $X$, if its complement $\sim \tilde{\tilde{A}} \in \tilde{\tilde{F}}$.

Proposition 3: Let $(X, \tilde{\widetilde{F}})$ be general type-2 fuzzy topological space over $X$ then the following conditions hold:

1) $\tilde{\tilde{\varnothing}}, \tilde{\tilde{X}}$ are type-2 fuzzy closed sets.

2) Arbitrary intersection of type-2 fuzzy closed sets is closed sets.

3) Finite union of type-2 fuzzy closed sets is closed sets.

Proof:

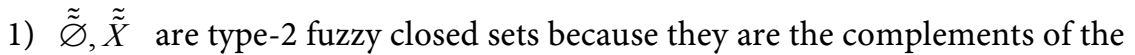
type-2 fuzzy open sets $\tilde{\tilde{\varnothing}}, \tilde{\tilde{X}}$ is respectively.

2) Let $\left\{\tilde{\tilde{A}}_{i}: i \in \mathbb{N}\right\}$ be an arbitrary collection of type-2 fuzzy closed sets, then

$$
\begin{aligned}
{\left[\cap_{i \in \mathbb{N}} \tilde{\tilde{A}}_{i}\right](x) } & =\sum_{x \in X} \sum_{u \in J_{x}^{u}} \frac{\wedge_{i \in \mathcal{N}}\left(f_{x}(u)\right)_{i}}{\wedge_{i \in \mathcal{N}}(u)_{i}} \\
& =\sum_{x \in X} \sum_{u \in J_{x}^{u}} \frac{\wedge_{i \in \mathcal{N}}\left(f_{x}(u)\right)_{i}}{1-\left(\vee_{i \in \mathcal{N}}(1-u)\right)_{i}}(\text { proposition 2.7 part 3) } \\
& =\left[\cup_{i \in \mathbb{N}} \sim \tilde{\tilde{A}}_{i}\right](x)
\end{aligned}
$$

since arbitrary union of type-2 fuzzy open sets are open $\left[\cup_{i \in \mathbb{N}} \sim \tilde{\tilde{A}}_{i}\right](x)$ is an open and $\left[\cap_{i \in \mathbb{N}} \tilde{\tilde{A}}_{i}\right](x)$ is a type-2 fuzzy closed sets.

3) If $\tilde{\tilde{A}}_{i}(i \in \mathbb{N})$ is type-2 fuzzy closed sets, then $\cup_{i \in \mathbb{N}} \tilde{\tilde{A}}_{i}$ is a type-2 fuzzy closed set, [finite intersection of type-2 fuzzy open sets are open].

Example 4: Let $X=\left\{x_{1}, x_{2}\right\}$ and let $\tilde{\tilde{A}}, \tilde{\tilde{\varnothing}}$ and $\tilde{\tilde{X}}$ be three type-2 fuzzy sets in $X$ which are

$$
\begin{aligned}
& \tilde{\tilde{\varnothing}}=\left(\left(x_{1}, 0\right), 1\right),\left(\left(x_{2}, 0\right), 1\right), \quad \tilde{\tilde{X}}=\left\{\left(\left(x_{1}, 1\right), 1\right),\left(\left(x_{2}, 1\right), 1\right)\right\} \\
& \tilde{\tilde{A}}=\left\{\left(\left(x_{1}, 0.8\right), 1\right),\left(\left(x_{1}, 0.6\right), 0.7\right),\left(\left(x_{1}, 0.3\right), 0.6\right),\right. \\
& \left.\left(\left(x_{2}, 0.8\right), 0.9\right),\left(\left(x_{2}, 0.5\right), 1\right),\left(\left(x_{2}, 0.4\right), 0.5\right)\right\} . \\
& \tilde{\tilde{\varnothing}} \cup \tilde{\tilde{X}} \text { for } x_{1}: \mu_{\tilde{\tilde{\chi}} \cup \tilde{X}}\left(x_{1}\right)=\frac{1 \wedge 1}{0 \vee 1} \Rightarrow=(1,1) \Rightarrow=\left\{\left(\left(x_{1}, 1\right), 1\right)\right\} \text {. } \\
& \tilde{\tilde{\varnothing}} \cup \tilde{\tilde{X}} \text { for } x_{2}: \mu_{\tilde{\varnothing} \cup \tilde{X}}\left(x_{2}\right)=\frac{1 \wedge 1}{0 \vee 1} \Rightarrow=(1,1) \Rightarrow=\left\{\left(\left(x_{2}, 1\right), 1\right)\right\} \text {. } \\
& \tilde{\tilde{\varnothing}} \cup \tilde{\tilde{X}}=\left\{\left(\left(x_{1}, 1\right), 1\right),\left(\left(x_{2}, 1\right), 1\right)\right\}=\tilde{\tilde{X}} \\
& \tilde{\tilde{\varnothing}} \cap \tilde{\tilde{X}} \text { for } x_{1}: \mu_{\tilde{\varnothing} \tilde{\tilde{X}} \tilde{\tilde{X}}}\left(x_{1}\right)=\frac{1 \wedge 1}{0 \wedge 1} \Rightarrow=(0,1) \Rightarrow=\left\{\left(\left(x_{1}, 0\right), 1\right)\right\} \text {. } \\
& \tilde{\tilde{\varnothing}} \cap \tilde{\tilde{X}} \text { for } x_{2}: \mu_{\tilde{\varnothing} \cap \tilde{\tilde{X}}}\left(x_{2}\right)=\frac{1 \wedge 1}{0 \wedge 1} \Rightarrow=(0,1) \Rightarrow=\left\{\left(\left(x_{2}, 0\right), 1\right)\right\} . \\
& \tilde{\tilde{\varnothing}} \cap \tilde{\tilde{X}}=\left\{\left(\left(x_{1}, 0\right), 1\right),\left(\left(x_{2}, 0\right), 1\right)\right\}=\tilde{\tilde{\varnothing}} \\
& \tilde{\varnothing} \cup \tilde{\tilde{A}} \text { for } x_{1}: \mu_{\tilde{\varnothing} \cup \tilde{\tilde{A}}}\left(x_{1}\right)=\frac{1 \wedge 1}{0 \vee 0.8}+\frac{1 \wedge 0.7}{0 \vee 0.6}+\frac{1 \wedge 0.6}{0 \vee 0.3} \\
& =\left\{\left(\left(x_{1}, 0.8\right), 1\right),\left(\left(x_{1}, 0.6\right), 0.7\right),\left(\left(x_{1}, 0.3\right), 0.6\right)\right\}
\end{aligned}
$$




$$
\begin{aligned}
& \tilde{\tilde{\varnothing}} \cup \tilde{\tilde{A}} \text { for } x_{2}: \mu_{\tilde{\tilde{\chi}} \cup \tilde{\tilde{A}}}\left(x_{2}\right)=\frac{1 \wedge 0.9}{0 \vee 0.8}+\frac{1 \wedge 1}{0 \vee 0.5}+\frac{1 \wedge 0.5}{0 \vee 0.4} \\
& =\left\{\left(\left(x_{2}, 0.8\right), 0.9\right),\left(\left(x_{2}, 0.5\right), 1\right),\left(\left(x_{2}, 0.4\right), 0.5\right)\right\} \\
& \tilde{\tilde{\varnothing}} \cup \tilde{\tilde{A}}=\left\{\left(\left(x_{1}, 0.8\right), 1\right),\left(\left(x_{1}, 0.6\right), 0.7\right),\left(\left(x_{1}, 0.3\right), 0.6\right)\right. \text {, } \\
& \left.\left(\left(x_{2}, 0.8\right), 0.9\right),\left(\left(x_{2}, 0.5\right), 1\right),\left(\left(x_{2}, 0.4\right), 0.5\right)\right\}=\tilde{\tilde{A}} \\
& \tilde{\tilde{\varnothing}} \cap \tilde{\tilde{A}} \text { for } x_{1}: \mu_{\tilde{\varnothing} \cap \tilde{\tilde{A}}}\left(x_{1}\right)=\frac{1 \wedge 1}{0 \wedge 0.8}+\frac{1 \wedge 0.7}{0 \wedge 0.6}+\frac{1 \wedge 0.6}{0 \wedge 0.3}=\frac{1}{0}+\frac{0.7}{0}+\frac{0.6}{0} \\
& =(0, \max \{1,0.7,0.6\}) \Rightarrow\left\{\left(\left(x_{1}, 0\right), 1\right)\right\} \text {, } \\
& \tilde{\tilde{\varnothing}} \cap \tilde{\tilde{A}} \text { for } x_{2}: \mu_{\tilde{\varnothing} \cap \tilde{\tilde{A}}}\left(x_{2}\right)=\frac{1 \wedge 0.9}{0 \wedge 0.8}+\frac{1 \wedge 1}{0 \wedge 0.5}+\frac{1 \wedge 0.5}{0 \wedge 0.4}=\frac{0.9}{0}+\frac{1}{0}+\frac{0.5}{0} \\
& =(0, \max \{0.9,1,0.5\}) \Rightarrow\left\{\left(\left(x_{2}, 0\right), 1\right)\right\} \text {, } \\
& \tilde{\tilde{\varnothing}} \cap \tilde{\tilde{A}}=\left\{\left(\left(x_{1}, 0\right), 1\right),\left(\left(x_{2}, 0\right), 1\right)\right\}=\tilde{\tilde{\varnothing}} \\
& \tilde{\tilde{A}} \cup \tilde{\tilde{X}} \text { for } x_{1}: \mu_{\tilde{\tilde{A}} \cup \tilde{X}}\left(x_{1}\right)=\frac{1 \wedge 1}{1 \vee 0.8}+\frac{1 \wedge 0.7}{1 \vee 0.6}+\frac{1 \wedge 0.6}{1 \vee 0.3}=\frac{1}{1}+\frac{0.7}{1}+\frac{0.6}{1} \\
& =(1, \max \{1,0.7,0.6\}) \Rightarrow\left\{\left(\left(x_{1}, 1\right), 1\right)\right\} \text {, } \\
& \tilde{\tilde{A}} \cup \tilde{\tilde{X}} \text { for } x_{2}: \mu_{\tilde{\tilde{A}} \cup \tilde{X}}\left(x_{2}\right)=\frac{1 \wedge 0.9}{1 \vee 0.8}+\frac{1 \wedge 1}{1 \vee 0.5}+\frac{1 \wedge 0.5}{1 \vee 0.4}=\frac{0.9}{1}+\frac{1}{1}+\frac{0.5}{1} \\
& =(1, \max \{1,0.9,0.5\}) \Rightarrow\left\{\left(\left(x_{2}, 1\right), 1\right)\right\} \\
& \tilde{\tilde{A}} \cup \tilde{\tilde{X}}=\tilde{\tilde{X}} \\
& \tilde{\tilde{A}} \cap \tilde{\tilde{X}} \text { for } x_{1}: \mu_{\tilde{\tilde{A}} \cap \tilde{\tilde{X}}}\left(x_{1}\right)=\frac{1 \wedge 1}{1 \wedge 0.8}+\frac{1 \wedge 0.7}{1 \wedge 0.6}+\frac{1 \wedge 0.6}{1 \wedge 0.3}=\frac{1}{0.8}+\frac{0.7}{0.6}+\frac{0.6}{0.3} \\
& =\left\{\left(\left(x_{1}, 0.8\right), 1\right),\left(\left(x_{1}, 0.6\right), 0.7\right),\left(\left(x_{1}, 0.3\right), 0.6\right)\right\} \\
& \tilde{\tilde{A}} \cap \tilde{\tilde{X}}=\left\{\left(\left(x_{1}, 0.8\right), 1\right),\left(\left(x_{1}, 0.6\right), 0.7\right),\left(\left(x_{1}, 0.3\right), 0.6\right),\right. \\
& \left.\left(\left(x_{2}, 0.8\right), 0.9\right),\left(\left(x_{2}, 0.5\right), 1\right),\left(\left(x_{2}, 0.4\right), 0.5\right)\right\}=\tilde{\tilde{A}}
\end{aligned}
$$

Then $\tilde{\tilde{F}}=\{\tilde{\tilde{X}}, \tilde{\varnothing}, \tilde{\tilde{A}}\}$ is general type-2 fuzzy topologies defined on $X$ and the pair $(X, \tilde{\tilde{F}})$ is called general type-2 fuzzy topological space over $X$, every member of $\tilde{\tilde{F}}$ is called type-2 fuzzy open sets.

Theorem 5: Let $\left\{\tilde{\tilde{\mathfrak{F}}}_{r}: r \in \mathbb{R}\right\}$ be a family of all general type-2 fuzzy topologies on $X$; then $\cap_{r \in \mathbb{R}} \tilde{\tilde{\mathfrak{F}}}_{r}$ is general type-2 fuzzy topologies on $X$.

proof: we must prove three conditions of topologies,

1) $\tilde{\tilde{\varnothing}}, \tilde{\tilde{X}} \in\left\{\tilde{\widetilde{\mathfrak{F}}}_{r}: r \in \mathbb{R}\right\} \Rightarrow \tilde{\tilde{\varnothing}}, \tilde{\tilde{X}} \in \cap_{r \in \mathbb{R}} \tilde{\widetilde{F}}_{r}$.

2) Let $\left\{\tilde{\tilde{A}}_{i}: i \in \mathbb{N}\right\} \subseteq \cap_{r \in \mathbb{R}} \tilde{\tilde{\mathcal{F}}}_{r}$, then $\tilde{\tilde{A}}_{i} \in \tilde{\tilde{\mathfrak{F}}}_{r}$ for all $i \in \mathbb{N}$ so thus $\cup_{i \in \mathbb{N}} \tilde{\tilde{A}}_{i} \in \cap_{r \in \mathbb{R}} \tilde{\tilde{\mathfrak{F}}}_{r}$.

3) Let $\tilde{\tilde{A}}, \tilde{\tilde{B}} \in \cap_{r \in \mathbb{R}} \tilde{\tilde{\mathfrak{F}}}_{r}$, then $\tilde{\tilde{A}}, \tilde{\tilde{B}} \in \tilde{\tilde{\mathfrak{F}}}_{r}$ and because $\tilde{\tilde{\mathfrak{F}}}_{r}$ are all general type-2 
fuzzy topologies $\tilde{\tilde{A}} \cap \tilde{\tilde{B}} \in \tilde{\tilde{F}}_{r}$ for all $r \in \mathbb{R}$, so $\tilde{\tilde{A}} \cap \tilde{\tilde{B}} \in \cap_{r \in \mathbb{R}} \tilde{\widetilde{\mathfrak{F}}}_{r}$.

Remark 6: Let $\left(X, \tilde{\widetilde{F}}_{1}\right)$ and $\left(X, \tilde{\widetilde{F}}_{2}\right)$ be two general type-2 fuzzy topological spaces over the same universe $X$ then $\left(X, \tilde{\widetilde{\mathfrak{F}}}_{1} \cup \tilde{\widetilde{\mathfrak{F}}}_{2}\right)$ need not be general type-2 fuzzy topological space over $X$, we can see that in example 3.7.

Example 7: Let $X=\left\{x_{1}, x_{2}\right\}$ and $\tilde{\widetilde{F}}_{1}=\{\tilde{\tilde{X}}, \tilde{\widetilde{\varnothing}}, \tilde{\tilde{A}}\}, \quad \tilde{\widetilde{F}}_{2}=\{\tilde{\tilde{X}}, \tilde{\widetilde{\varnothing}}, \tilde{\tilde{B}}\}$ be two general type-2fuzzy topologies defined on $X$ where $\tilde{\tilde{A}}, \tilde{\tilde{B}}, \tilde{\tilde{\varnothing}}$ and $\tilde{\tilde{X}}$ defined as follows: $\tilde{\tilde{\varnothing}}=\left\{\left(\left(x_{1}, 0\right), 1\right),\left(\left(x_{2}, 0\right), 1\right)\right\}$,

$$
\begin{gathered}
\tilde{\tilde{X}}=\left\{\left(\left(x_{1}, 1\right), 1\right),\left(\left(x_{2}, 1\right), 1\right)\right\} \\
\tilde{\tilde{A}}=\left\{\left(\left(x_{1}, 0.8\right), 1\right),\left(\left(x_{1}, 0.6\right), 0.7\right),\left(\left(x_{1}, 0.3\right), 0.6\right),\right. \\
\left.\left(\left(x_{2}, 0.8\right), 0.9\right),\left(\left(x_{2}, 0.5\right), 1\right),\left(\left(x_{2}, 0.4\right), 0.5\right)\right\} . \\
\tilde{\tilde{B}}=\left\{\left(\left(x_{1}, 0.5\right), 1\right),\left(\left(x_{1}, 0.6\right), 0.2\right),\left(\left(x_{2}, 0.3\right), 0.7\right),\left(\left(x_{2}, 0.9\right), 1\right)\right\} .
\end{gathered}
$$

Let $\tilde{\tilde{\mathfrak{F}}}_{1} \cup \tilde{\tilde{\mathfrak{F}}}_{2}=\{\tilde{\tilde{\varnothing}}, \tilde{\tilde{X}}, \tilde{\tilde{A}}, \tilde{\tilde{B}}\}$ so $\left(X, \tilde{\tilde{\mathfrak{F}}}_{1} \cup \tilde{\tilde{\mathfrak{F}}}_{2}\right)$ is not general type-2 fuzzy topological space over $X$ since $\tilde{\tilde{A}} \cap \tilde{\tilde{B}} \notin \tilde{\tilde{\mathfrak{F}}}_{1} \cup \tilde{\tilde{\mathfrak{F}}}_{2}$.

Definition 8: Let $(X, \tilde{\tilde{F}})$ be general type-2 fuzzy topological space over $X$ and let $\tilde{\tilde{A}}$ be type-2 fuzzy set over $X$. Then the type-2 fuzzy interior of $\tilde{\tilde{A}}$, denoted by $\operatorname{int}(\tilde{\tilde{A}})$, is defined as the union of all type-2 fuzzy open sets contained in $\tilde{\tilde{A}}$. That is,

$\operatorname{int}(\tilde{\tilde{A}})=\cup\left\{\tilde{\tilde{G}}_{i}: \tilde{\tilde{G}}_{i}\right.$ type-2 fuzzy open sets in $\left.X, \tilde{\tilde{G}}_{i} \subseteq \tilde{\tilde{A}}, i \in \mathbb{N}\right\}, \quad \operatorname{int}(\tilde{\tilde{A}})$ is the largest type-2 fuzzy open set contained in $\tilde{\tilde{A}}$.

Theorem 9: Let $(X, \tilde{\tilde{F}})$ be general type-2 fuzzy topological space over $X$, and let $\tilde{\tilde{A}}, \tilde{\tilde{B}}$ be two type-2 fuzzy sets in $X$. Then

1) $\operatorname{int}(\tilde{\tilde{\varnothing}})=\tilde{\tilde{\varnothing}}$ and $\operatorname{int}(\tilde{\tilde{X}})=\tilde{\tilde{X}}$.

2) $\operatorname{int}(\tilde{\tilde{A}}) \subseteq \tilde{\tilde{A}}$.

3) $\tilde{\tilde{A}}$ is type-2 fuzzy open set if and only if $\operatorname{int}(\tilde{\tilde{A}})=\tilde{\tilde{A}}$.

4) $\operatorname{int}(\operatorname{int}(\tilde{\tilde{A}}))=\operatorname{int}(\tilde{\tilde{A}})$.

5) $\tilde{\tilde{A}} \subseteq \tilde{\tilde{B}} \rightarrow \operatorname{int}(\tilde{\tilde{A}}) \subseteq \operatorname{int}(\tilde{\tilde{B}})$.

6) $\operatorname{int}(\tilde{\tilde{A}} \cap \tilde{\tilde{B}})=\operatorname{int}(\tilde{\tilde{A}}) \cap \operatorname{int}(\tilde{\tilde{B}})$.

Proof:

1) $\operatorname{int}(\tilde{\tilde{A}})=\cup\left\{\tilde{\tilde{G}}_{i}: \tilde{\tilde{G}}_{i}\right.$ type-2 fuzzy open sets in $\left.X, \tilde{\tilde{G}}_{i} \subseteq \tilde{\tilde{A}}, i \in \mathbb{N}\right\}, \quad \tilde{\tilde{\varnothing}} \quad$ is type-2 fuzzy open set in $\tilde{\tilde{F}}$ and $\tilde{\tilde{\varnothing}} \subseteq \tilde{\tilde{\varnothing}} \Rightarrow \operatorname{int}(\tilde{\tilde{\varnothing}})=\tilde{\tilde{\varnothing}}$. 
Now to prove $\operatorname{int}(\tilde{\tilde{X}})=\tilde{\tilde{X}}$,

$\operatorname{int}(\tilde{\tilde{X}})=\cup\left\{\tilde{\tilde{G}}_{i}: \tilde{\tilde{G}}_{i}\right.$ type-2 fuzzy open sets in $\left.X, \tilde{\tilde{G}}_{i} \subseteq \tilde{\tilde{X}}, i \in \mathbb{N}\right\}, \quad \tilde{\tilde{X}}$ is type-2

fuzzy open set in $\tilde{\tilde{F}}$ and $\tilde{\tilde{X}} \subseteq \tilde{\tilde{X}} \Rightarrow \operatorname{int}(\tilde{\tilde{X}})=\tilde{\tilde{X}}$.

2) To prove $\operatorname{int}(\tilde{\tilde{A}}) \subseteq \tilde{\tilde{A}}$, since

$\operatorname{int}(\tilde{\tilde{A}})=\cup\left\{\tilde{\tilde{G}}_{i}: \tilde{\tilde{G}}_{i}\right.$ type-2 fuzzy open sets in $\left.X, \tilde{\tilde{G}}_{i} \subseteq \tilde{\tilde{A}}, i \in \mathbb{N}\right\}$, such that $\tilde{\tilde{G}}_{i} \subseteq \tilde{\tilde{A}}$

that is $\tilde{\tilde{A}}$ is type-2 membership function $\mu_{\tilde{\tilde{A}}}(x, u)$ where $x \in X$ and $u \in J_{X} \subseteq[0,1]$ less than a type-2 membership function $\mu_{\tilde{\tilde{G}}_{i}}(x, u)$ where $x \in X$ and $w \in J_{X} \subseteq[0,1]$ such that $w \leq u$ and $\mu_{\tilde{\tilde{G}}_{i}}(x, u) \leq \mu_{\tilde{\tilde{A}}}(x, u)$, $\sup \left\{\mu_{\tilde{\tilde{G}}_{i}}(x, u) \leq \mu_{\tilde{\tilde{A}}}(x, u), w \leq u\right\} \quad$ hence $\cup \tilde{\tilde{G}}_{i} \subseteq \tilde{\tilde{A}} \Rightarrow \cup \tilde{\tilde{G}}_{i} \subseteq \operatorname{int}(\tilde{\tilde{A}})$, therefore $\operatorname{int}(\tilde{\tilde{A}}) \subseteq \tilde{\tilde{A}}$.

3) If $\tilde{\tilde{A}}$ is type-2 fuzzy open set, then $\tilde{\tilde{A}} \subseteq \operatorname{int}(\tilde{\tilde{A}})$, but $\operatorname{int}(\tilde{\tilde{A}}) \subseteq \tilde{\tilde{A}}$ from part (2), hence $\operatorname{int}(\tilde{\tilde{A}})=\tilde{\tilde{A}}$.

4) $\operatorname{int}(\tilde{\tilde{A}})$ is a type-2 fuzzy open set and from part (3) we have $\operatorname{int}(\operatorname{int}(\tilde{\tilde{A}}))=\operatorname{int}(\tilde{\tilde{A}})$

5) If $\tilde{\tilde{A}} \subseteq \tilde{\tilde{B}}$ and from $\operatorname{part}(2) \operatorname{int}(\tilde{\tilde{A}}) \subseteq \tilde{\tilde{A}}$, int $(\tilde{\tilde{B}}) \subseteq \tilde{\tilde{B}}$, then $\operatorname{int}(\tilde{\tilde{A}}) \subseteq \tilde{\tilde{A}} \subseteq \tilde{\tilde{B}}$. Therefore $\operatorname{int}(\tilde{\tilde{A}}) \subseteq \tilde{\tilde{B}}$ and $\operatorname{int}(\tilde{\tilde{A}})$ is a type-2 fuzzy open set contained in $\tilde{\tilde{B}}$, so $\operatorname{int}(\tilde{\tilde{A}}) \subseteq \operatorname{int}(\tilde{\tilde{B}})$.

6) Because $(\tilde{\tilde{A}} \cap \tilde{\tilde{B}}) \subseteq \tilde{\tilde{A}}$ and $(\tilde{\tilde{A}} \cap \tilde{\tilde{B}}) \subseteq \tilde{\tilde{B}}$, from part (5)

$\operatorname{int}(\tilde{\tilde{A}} \cap \tilde{\tilde{B}}) \subseteq \operatorname{int}(\tilde{\tilde{A}})$ and $\operatorname{int}(\tilde{\tilde{A}} \cap \tilde{\tilde{B}}) \subseteq \operatorname{int}(\tilde{\tilde{B}})$, thus

$\operatorname{int}(\tilde{\tilde{A}} \cap \tilde{\tilde{B}}) \subseteq \operatorname{int}(\tilde{\tilde{A}}) \cap \operatorname{int}(\tilde{\tilde{B}})$, since $\operatorname{int}(\tilde{\tilde{A}} \cap \tilde{\tilde{B}}) \subseteq \tilde{\tilde{A}} \cap \tilde{\tilde{B}}$, so

$\operatorname{int}(\operatorname{int}(\tilde{\tilde{A}})) \cap \operatorname{int}(\tilde{\tilde{B}}) \subseteq(\tilde{\tilde{A}} \cap \tilde{\tilde{B}})$ from $\operatorname{part}(5)$ but $\operatorname{int}(\tilde{\tilde{A}}) \cap \operatorname{int}(\tilde{\tilde{B}})$ is a type-2 fuzzy open sets then $\operatorname{int}(\operatorname{int}(\tilde{\tilde{A}})) \cap \operatorname{int}(\tilde{\tilde{B}}) \subseteq \operatorname{int}(\tilde{\tilde{A}} \cap \tilde{\tilde{B}})$ from $\operatorname{part}(3)$.Hence $\operatorname{int}(\tilde{\tilde{A}} \cap \tilde{\tilde{B}})=\operatorname{int}(\tilde{\tilde{A}}) \cap \operatorname{int}(\tilde{\tilde{B}})$.

Definition 10: Let $(X, \tilde{\tilde{F}})$ be general type-2 fuzzy topological space over $\tilde{\tilde{X}}$ and let $\tilde{\tilde{A}}$ be type-2 fuzzy set over $X$. Then the type-2 fuzzy closure of $\tilde{\tilde{A}}$, denoted by $c l(\tilde{\tilde{A}})$, is defined as the intersection of all type-2 fuzzy closed sets containing $\tilde{\tilde{A}}$. That is

$$
c l(\tilde{\tilde{A}})=\cap\left\{\tilde{\tilde{M}}_{i}: \tilde{\tilde{M}}_{i} \text { type-2 fuzzy closed sets in } X, \tilde{\tilde{A}} \subseteq \tilde{\tilde{M}}_{i}, i \in \mathbb{N}\right\},
$$


$\operatorname{cl}(\tilde{\tilde{A}})$ is the smallest type-2 fuzzy closed set containing $\tilde{\tilde{A}}$.

Theorem 11: Let $(X, \tilde{\tilde{F}})$ be general type-2 fuzzy topological space over $X$, and let $\tilde{\tilde{A}}, \tilde{\tilde{B}}$ be two type-2 fuzzy sets in $X$. Then

1) $\operatorname{cl}(\tilde{\tilde{\varnothing}})=\tilde{\tilde{\varnothing}}$ and $\operatorname{cl}(\tilde{\tilde{X}})=\tilde{\tilde{X}}$.

2) $\tilde{\tilde{A}} \subseteq \operatorname{cl}(\tilde{\tilde{A}})$.

3) $\tilde{\tilde{A}}$ is type-2 fuzzy closed set if and only if $\operatorname{cl}(\tilde{\tilde{A}})=\tilde{\tilde{A}}$.

4) $\operatorname{cl}(\operatorname{cl}(\tilde{\tilde{A}}))=\operatorname{cl}(\tilde{\tilde{A}})$.

5) $\quad \tilde{\tilde{A}} \subseteq \tilde{\tilde{B}} \rightarrow \operatorname{cl}(\tilde{\tilde{A}}) \subseteq \operatorname{cl}(\tilde{\tilde{B}})$.

6) $\operatorname{cl}(\tilde{\tilde{A}} \cap \tilde{\tilde{B}})=\operatorname{cl}(\tilde{\tilde{A}}) \cap \operatorname{cl}(\tilde{\tilde{B}})$.

Proof: The proof this theorem similar to the proof of theorem 3.7.

Definition 12: Let $(X, \tilde{\tilde{F}})$ be a general type-2 fuzzy topological space over $X$ and $\tilde{\tilde{N}} \subseteq \tilde{\tilde{F}}$. Then is said to be a neighborhood or nbhd for short, of a type-2 fuzzy set $\tilde{\tilde{A}}$ if there exist a type-2 fuzzy open set $\tilde{\tilde{W}}$ such that $\tilde{\tilde{A}} \subseteq \tilde{\tilde{W}} \subseteq \tilde{\tilde{N}}$.

Proposition 13: A type-2 fuzzy set $\tilde{\tilde{A}}$ is open if and only if for each type-2 fuzzy set $\tilde{\tilde{B}}$ contained in $\tilde{\tilde{A}}, \tilde{\tilde{A}}$ is a neighborhood of $\tilde{\tilde{B}}$.

Proof: If $\tilde{\tilde{A}}$ is open and $\tilde{\tilde{B}} \subseteq \tilde{\tilde{A}}$ then $\tilde{\tilde{A}}$ is a neighborhood of $\tilde{\tilde{B}}$. Conversely, since $\tilde{\tilde{A}} \subseteq \tilde{\tilde{A}}$, there exists a type-2 fuzzy open set $\tilde{\tilde{W}}$ such that $\tilde{\tilde{A}} \subseteq \tilde{\tilde{W}} \subseteq \tilde{\tilde{A}}$. Hence $\tilde{\tilde{A}}=\tilde{\tilde{W}}$ and $\tilde{\tilde{A}}$ is open.

Definition 14: Let $(X, \tilde{\tilde{F}})$ be a general type-2 fuzzy topological space over $X$ and $\tilde{\tilde{\mathfrak{B}}}$ be a subfamily of $\tilde{\tilde{\mathfrak{F}}}$. If every member of $\tilde{\tilde{\mathfrak{F}}}$ can be written as the type-2 fuzzy union of some members of $\tilde{\mathfrak{\mathfrak { B }}}$, then $\tilde{\tilde{\mathfrak{B}}}$ is called a type-2 fuzzy base for the general type-2 fuzzy topology $\tilde{\tilde{\mathfrak{F}}}$. We can see that if $\tilde{\tilde{\mathfrak{B}}}$ be type-2 fuzzy base for $\tilde{\tilde{\mathfrak{F}}}$ then $\tilde{\tilde{\mathfrak{F}}}$ equals the collection of type-2 fuzzy unions of elements of $\tilde{\tilde{\mathfrak{B}}}$.

Definition 15: Let $(X, \tilde{\tilde{F}})$ and $(Y, \tilde{\tilde{S}})$ be two general type-2 fuzzy topological space.The general type-2 fuzzy topological space $Y$ is called a subspace of the general type-2 fuzzy topological space $X$ if $Y \subseteq X$ and the open subsets of $Y$ are precisely of the form $\tilde{\tilde{\mathcal{F}}}_{\tilde{\tilde{Y}}}=\{\tilde{\tilde{\mathcal{Y}}}=\tilde{\tilde{Y}} \cap \tilde{\tilde{\mathcal{X}}}: \tilde{\tilde{\mathcal{X}}} \in \tilde{\tilde{\mathcal{F}}}\}$. Here we may say that each open subset $\tilde{\tilde{\mathcal{Y}}}$ of $Y$ is the restriction to $\tilde{\tilde{\mathcal{Y}}}$ of an open subset $\tilde{\tilde{\mathcal{X}}}$ of $X$. That is, $(Y, \tilde{\tilde{S}})$ is called a subspace of $(X, \tilde{\tilde{F}})$ if the type-2 fuzzy open sets of $Y$ are the type-2 fuzzy intersection of open sets of $X$ with $\tilde{\tilde{\mathcal{Y}}}$.

\section{Conclusion}

The main purpose of this paper is to introduce a new concept in fuzzy set theory, namely that of general type-2 fuzzy topological space. On the other hand, type-2 fuzzy set is a kind of abstract theory of mathematics. First, we present definition 
and properties of this set before introducing definition of general type-2 fuzzy topological space with the structural properties such as open sets, closed sets, interior, closure and neighborhoods in general type-2 fuzzy set topological spaces and some definitions of a type- 2 fuzzy base and subspace of general type- 2 fuzzy sets.

\section{Acknowledgements}

Great thanks to all those who helped us in accomplishing this research especially Prof. Dr. Kamal El-saady and Prof. Dr. Sherif Abuelenin from Egypt for us as well as all the workers in the magazine.

\section{Conflicts of Interest}

The authors declare no conflicts of interest regarding the publication of this paper.

\section{References}

[1] Zadeh, L.A. (1965) Fuzzy Sets. Information and Control, 8, 338-353. https://doi.org/10.1016/S0019-9958(65)90241-X

[2] Zhang, Z. (2013) On Characterization of Generalized Interval Type-2 Fuzzy Rough Sets. Information Sciences, 219, 124-150. https://doi.org/10.1016/j.ins.2012.07.013

[3] Chang, C.L. (1968) Fuzzy Topological Space. The Journal of Mathematical Analysis and Applications, 24, 182-190. https://doi.org/10.1016/0022-247X(68)90057-7

[4] Karnik, N.N. and Mendel, J.M. (2001) Operations on Type-2 Fuzzy Sets. Fuzzy Sets and Systems, 122, 327-348. https://doi.org/10.1016/S0165-0114(00)00079-8

[5] Mizumoto, M. and Tanaka, K. (1976) Some Properties of Fuzzy Sets of Type-2. Information and Control, 31, 312-340. https://doi.org/10.1016/S0019-9958(76)80011-3

[6] Mendel, J.M. (2001) Uncertain Rule-Based Fuzzy Logic Systems: Introduction and New Directions. Prentice-Hall, Upper Saddle River, NJ.

[7] Mendel, J.M. and John, R.I. (2002) Type-2 Fuzzy Sets Made Simple. IEEE Transactions on Fuzzy Systems, 10, 117-127. https://doi.org/10.1109/91.995115

[8] Zhao, T. and Wei, Z. (2015) On Characterization of Rough Type-2 Fuzzy Sets. Mathematical Problems in Engineering, 2016, 13.

[9] Zadeh, L.A. (1975) The Concept of a Linguistic Variable and Its Application to Approximate Reasoning-1. Information Sciences, 8, 199-249. https://doi.org/10.1016/0020-0255(75)90036-5 